\title{
VII. A necessary modification of Ohm's law
}

\section{Fernando Sanford}

To cite this article: Fernando Sanford (1893) VII. A necessary modification of Ohm's law , Philosophical Magazine Series 5, 35:212, 65-67, DOI: 10.1080/14786449308620381

To link to this article: http://dx.doi.org/10.1080/14786449308620381

$$
\text { 曲 Published online: } 08 \text { May } 2009 .
$$

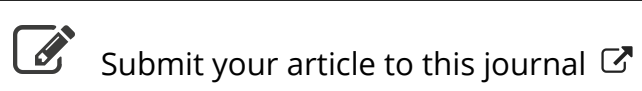

\footnotetext{
Џll Article views: 2
}

Q View related articles $\longleftarrow$ 
VII. A necessany Modification of Ohm's Law. By Fernando Sanford*.

[Plate III.]

$\mathrm{D}^{\mathrm{v}}$ URING the past year I have been engaged in some investigations which seem to call in question the validity of Ohm's law, by showing that the resistance of a metallic conductor varies with the nature of the dielectric in its field of force. For the purpose of determining if this be the case, I have made a large number of measurements of the resistance of a copper wire in various dielectrics, and have found that in several cases the change of resistance, both in liquid and gaseous dielectrics, is very marked.

The apparatus used consists of a copper tube about four feet long and one inch in internal diameter, closed with copper plates at the end, and having a copper wire 1 millim. in diameter stretched through its centre and fastened by means of a binding-screw to the centre of one end plate, while it passes through an insulating-plug in the centre of the other end plate. The tube is provided with stopcocks at the ends for filling and emptying, and with an opening in the side for inserting a thermometer. The current, which was always between the extremes of five and eight milliamperes, was passed one way through the tube and back through the wire, so that the whole dielectric in its field could be changed at will. The measurements were made by means of a Wheat stone's bridge with arms of $1: 1000$, and a sensitive galvanometer. A change of resistance of $0 \cdot 1 \mathrm{ohm}$ in the box, which corresponded to $.0001 \mathrm{ohm}$ in the tube and wire, produced a very roticeable deflexion of the galvanometer-needle. The measurements were accordingly estimated with a fair degree of accuracy to $00001 \mathrm{ohm}$.

The resistance of the tube and wire was measured in air at different temperatures through a range of about ten degrees Centigrade, and a curve was plotted, using the temperatures as abscissæ and the resistances as ordinates. This curve did not depart appreciably from a straight line. The dielectric to be tested was then poured into the tube, a set of measurements was made in it through the same range of temperatures, the dielectric drawn out, the tube cleaned and dried, and a new set of measurements made in air. This was repeated several times, until it was certain that after each change of dielectrics the resistance returned to the same value which it had previously shown in the same dielectric. In the case of air and petroleum this comparison was carried on for a month, the dielectrics in the meantime being changed five times, and

* Communicated by the Author.

Phil. Mag. S. 5. Vol, 35. No. 212. Jan. 1893. 
the apparatus being allowed to stand untouched for two weeks to make sure that none of the constants were changing, and in the whole series not a single measurement in either dielectric fell upon or beyond the curve made for the other dielectric (see Pl. III. fig. 1). The resistance of the wire in petroleum was shown to be about $\cdot 00006 \mathrm{ohm}$ less than in air ; and as the whole resistance of the tube and wire averaged about 0335 $\mathrm{ohm}$, this difference corresponded to about 18 of one per cent. of the whole resistance.

Regarding the conductivity of the wire in air as unity, its conductivity in the liquid dielectrics tested was as follows :-

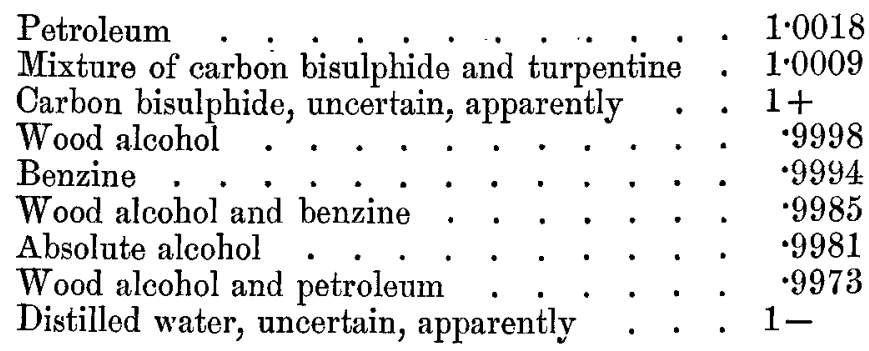

It will be seen that when two dielectrics which do not seem to mix with each other were used together the resistance of the wire was greatly increased, as in the case of wood alcohol and benzine, and wood alcohol and petroleum. That these liquids do diffuse into each other was shown by measuring the index of refraction of the two components before and after they had stood in contact with each other, and in every case a change was observed.

A similar change of resistance was noticed when the tube contained different gaseous dielectrics. This was first observed when the burning gas used in the laboratory was allowed to enter the tube. This gas was made from gasoline by a machine on the ground, and consisted of the vapour of gasoline mixed with air. A series of measurements was made in this gas and in air, the measurements in air being made both before and after those made in the gas. Of the seventeen measurements with the wire in the gas, only one was as much as ${ }^{\circ} 00001 \mathrm{ohm}$ from the curve drawn, while the nearest distance of any gas measurement to the air-curve was $\cdot 00004$ ohm, and the average distance of the gas points from the air-curve was $000058 \mathrm{ohm}$. From these measurements the resistance of the wire in gasoline burning gas was 1.0017 times its resistance in air (see fig. 2).

The other vapours used were those of volatile liquids, a small quantity of the liquid being poured into the tube and allowed to evaporate. Taking, as before, the conductivity of 


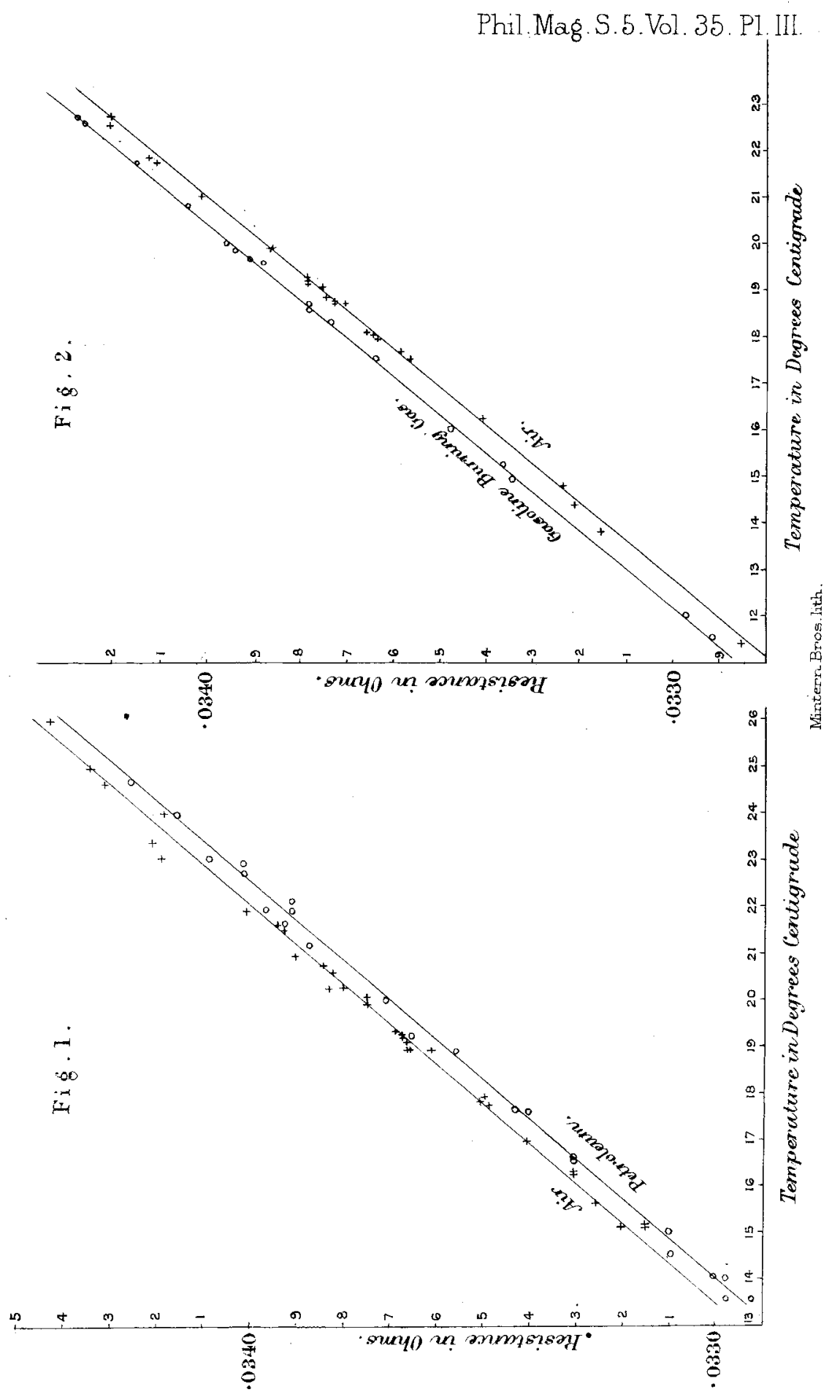


the wire in air as unity, the conductivities in the vapours tested were approximately as follows :-

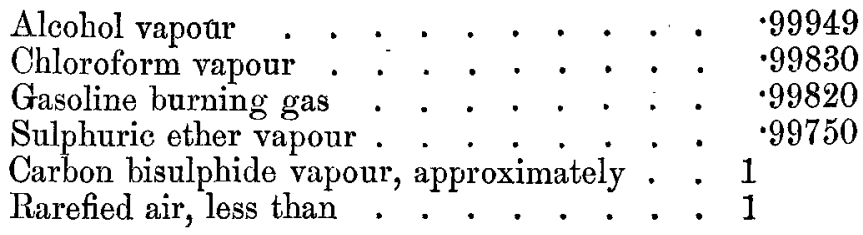

Some of the phenomena observed made it seem probable that only that part of the dielectric in direct contact with the wire was concerned in this change of resistarice. For example, when the petroleum was poured out of the tube and a measurement made before it had drained off and evaporated, the resistance observed was the same as when the tube was filled with petroleum, and it was only after the tube had been carefully drained out and dried that the resistance returned to its former value in air. In the case of sulphuric ether vapour this phenomenon was very marked. It was only after the. tube had been washed out with alcohol one or more times, and. had been dried out by drawing a current of air through it for several hours, that the resistance returned to its former value.

I am now engaged in investigating the same phenomenon, using a silver wire of the same size as the copper wire used in last year's experiments. In general, the difference in resistance with the dielectries above mentioned is less marked than in the case of the copper wire formerly used, but it is still noticeable. As in the case of the copper wire, the greatest variation yet noticed was when the tube was filled with sulphuric ether vapour, but with the silver wire the resistance is decreased in the ether vapour, while with the copper wire it was increased. 'The same difficulty in removing the effect of the ether vapour also appears with the silver wire, in which case it was only after repeated washings with alcohol that the resistance of the wire returned to its former value.

I havo thus far been unable to find with what other properties of the dielectrics these phenomena are correlated, but it seems clear that hereafter Ohm's Law will have to be so modified as to take account of the dielectric surrounding a conductor, as well as the nature and temperature of the conductor itself.

The measurements upon which the above conclusions are based are given in full in a paper entitled "Some Observations upon the Conductivity of a Copper Wire in various Dielectries," which is now being published by the Leland Stanford Jr. University.

Palo Alto, California,

October 26, 1892. 